\title{
EDITORIAL
}

\section{Rhinoviruses: markers of, or causative for, recurrent wheeze and asthma?}

\author{
E. Kieninger and N. Regamey
}

$\mathbf{R}$ hinovirus (RV) infections occur early and recurrently in life and impose a large burden of disease in infants and young children [1-3]. RVs are the most frequent pathogens detected during both upper and lower respiratory tract infections and are associated with a large spectrum of clinical outcomes in this age group $[4,5]$. In the very young, they are one of the leading agents of bronchiolitis, second only to respiratory syncytial virus (RSV), and in children aged $>12$ months of age they are predominant pathogens associated with wheezing episodes [6-8]. In addition to their impact on short-term morbidity, RVs have been shown to represent an important pathogenic factor in the development of recurrent wheeze and asthma; a role which is yet not fully understood [9-12]. Several studies have been conducted over the past few years evaluating the influence of RV infections in early life on the development and incidence of wheezing and asthma [13-17]. They have clearly demonstrated that RV-associated wheezing is a risk factor for asthma development, and that approximately one third of infants with recurrent RV-induced wheezing illnesses go on to develop asthma. Unfortunately, most of these studies have been conducted in infants at high risk for atopy and asthma and not in unselected populations, or have included hospitalised children of a wide age range. These are significant limitations in the understanding of the respective influences of virus infections, atopy, age and maturity of the immune system on asthma development on a population level.

In this issue of the European Respiratory Journal, MidULLA et al. [18] present a prospective, single-centre study in which they assessed possible risk factors for recurrent wheezing during a 1-yr followup in $>300$ infants aged $<12$ months hospitalised for their first episode of bronchiolitis. They found that RV-induced bronchiolitis (OR 3.3, 95\% CI 1.0-11.1) and a positive family history for asthma (OR 2.5, 95\% CI 1.2-4.9) were the strongest independent risk factors for recurrent wheezing and, therefore, concluded that a nasal washing positive for RV in infants hospitalised for bronchiolitis can possibly predict infants prone to the development of recurrent wheezing. These results concur well with a very recently published Finnish study in the European Respiratory Journal [19], in which children $<6$ months of age hospitalised for bronchiolitis were followed at 6 yrs of age, demonstrating that the risk of asthma was

Division of Respiratory Medicine, Dept of Paediatrics, University Children's Hospital of Berne, Berne, Switzerland.

CORRESPONDENCE: N. Regamey, Division of Respiratory Medicine, Dept of Paediatrics, University Children's Hospital of Bern, Inselspital, Freiburgstrasse, 3010 Berne, Switzerland. E-mail: Nicolas.Regamey@insel.ch lower after RSV bronchiolitis ( $8 \%$ ) than after bronchiolitis caused by other viruses $(24 \%)$. It will be interesting to see whether RVinduced bronchiolitis in the cohort of MiduLLA et al. [18] remains a risk factor for the development of asthma at school age.

One of the strengths of the study by MidulLA et al. [18] is that the authors investigated a clinically homogenous and well-characterised cohort of unselected infants. They included infants $<12$ months of age with a first episode of bronchiolitis, which was defined as an upper respiratory tract infection followed by acute onset of respiratory distress with cough, tachypnoea, retractions and diffuse crackles on auscultation. Thus, they avoided the pitfall of using wheezing as an entry criterion when the study outcome is also wheeze. Bronchiolitis is a clinical diagnosis without common international definition, which makes comparisons between studies, and especially assessment of factors, such as those predisposing to the development of asthma or predicting response to treatment, difficult [8]. Whilst in Europe the presence of inspiratory crackles on auscultation is regarded as an important definition criterion for bronchiolitis, and an upper limit of age of 12 months is usually chosen, the definition is applied more broadly to a first episode of acute viral wheeze within the first 2 yrs of life in North America [20]. Thus, early presentations of asthma are likely to overlap with the North American definition of bronchiolitis, which has major implications with regard to the understanding of the pathophysiology of asthma, but also with regard to treatment responses, for instance to steroids or inhalation with hypertonic saline [21, 22]. Therefore, a European Respiratory Society/American Thoracic Society Task Force on the definition and treatment of bronchiolitis is imperatively needed to address these issues.

The study by MidulLA et al. [18] also has shortcomings, which have to be considered when interpreting their findings. Besides the fact that the authors relied on parentally reported recurrent wheeze, which is at best imperfect [23], the detection rate of RV in their study was low (only 24 out of 313 infants), and as such the estimates of association were rather weak, with wide confidence intervals and only borderline statistical significances. In addition, there was a significant drop-out rate, with a high RV detection rate in the sub-cohort that was lost due to follow-up (five out of 51 infants). A few more RV-positive infants in the non-wheezing group would have made the study results no longer significant.

What still remains unanswered is the question as to whether RVs are directly involved in the development of childhood wheeze and asthma or whether they only represent a proxy for infants prone to developing obstructive lung diseases. These two scenarios are not mutually exclusive, but the fact that only some 
infants with RV-induced bronchiolitis go on to develop recurrent wheezing and/or asthma later in life suggests that other than viral factors must play a role in this process. Airway dysfunction could be a direct consequence of RV infections in early life, for instance through damage to the airway epithelium and the initiation of inflammatory and remodelling processes $[10,12]$. As the clinical presentation, dynamic of infection and immune responses differ according to rhinovirus species [24], it could be speculated that their respective influence on the development of recurrent wheeze and asthma varies. With respect to this, it would have been interesting to investigate the effects of the recently described RV-C species [25] in the study by Midulla et al. [18], but numbers were too small for such analysis. Host factors influencing susceptibility towards RV infections are numerous and include genetic factors [26], reduced pre-morbid lung function [27], impaired immune responses towards viruses [28, 29] and atopy $[13,14]$. The interplay of these factors is complex but most studies, including the current study by MiduLLA et al. [18], suggest that the highest risk for developing asthma is observed in children having both recurrent viral infections during infancy and atopic features, such as atopic dermatitis, early sensitisation to food or inhaled allergens, eosinophilia or a maternal history of allergy. Additionally, the timing and the frequency of RV infections with regard to the individual phase of immune development also seem to play an important role in this process. As infancy is a period of profound growth and development of the pulmonary and immune system, RV infections and associated inflammatory and remodelling processes occur during a vulnerable phase of ongoing developmental processes, which might be thus interrupted and/or disrupted [30].

Taken together, viral aetiology, host susceptibility (in particular allergic predisposition and sensitisation), and illness severity, timing and frequency all might contribute as synergistic factors to the risk of developing asthma. To disentangle these complex interactions, carefully conducted longitudinal and prospective population-based studies in unselected healthy infants are needed. They may allow us to more precisely define the relationships and underlying mechanisms linking RV infections in early life and development of recurrent wheeze and asthma in childhood, and ultimately lead to better targeted preventive and therapeutic measures for these conditions.

\section{STATEMENT OF INTEREST}

None declared.

\section{REFERENCES}

1 Regamey N, Kaiser L. Rhinovirus infections in infants: is respiratory syncytial virus ready for the challenge? Eur Respir J 2008; 32: 249-251.

2 Miller EK, Lu X, Erdman DD, et al. Rhinovirus-associated hospitalizations in young children. J Infect Dis 2007; 195: 773-781.

3 Jartti T, Lee WM, Pappas T, et al. Serial viral infections in infants with recurrent respiratory illnesses. Eur Respir J 2008; 32: 314-320.

4 Regamey N, Kaiser L, Roiha HL, et al. Viral etiology of acute respiratory infections with cough in infancy: a community-based birth cohort study. Pediatr Infect Dis J 2008; 27: 100-105.

5 Papadopoulos NG, Moustaki M, Tsolia M, et al. Association of rhinovirus infection with increased disease severity in acute bronchiolitis. Am J Respir Crit Care Med 2002; 165: 1285-1289.

6 Rakes GP, Arruda E, Ingram JM, et al. Rhinovirus and respiratory syncytial virus in wheezing children requiring emergency care. IgE and eosinophil analyses. Am J Respir Crit Care Med 1999; 159: 785-790.
7 Midulla F, Scagnolari C, Bonci E, et al. Respiratory syncytial virus, human bocavirus and rhinovirus bronchiolitis in infants. Arch Dis Child 2010; 95: 35-41.

8 Jartti T, Lehtinen P, Vuorinen $\mathrm{T}$, et al. Bronchiolitis: age and previous wheezing episodes are linked to viral etiology and atopic characteristics. Pediatr Infect Dis J 2009; 28: 311-317.

9 Jartti T, Korppi M. Rhinovirus-induced bronchiolitis and asthma development. Pediatr Allergy Immunol 2011; 22: 350-355.

10 Gavala ML, Bertics PJ, Gern JE. Rhinoviruses, allergic inflammation, and asthma. Immunol Rev 2011; 242: 69-90.

11 Jackson DJ. The role of rhinovirus infections in the development of early childhood asthma. Curr Opin Allergy Clin Immunol 2010; 10: 133-138.

12 Gern JE. Rhinovirus and the initiation of asthma. Curr Opin Allergy Clin Immunol 2009; 9: 73-78.

13 Jackson DJ, Gangnon RE, Evans MD, et al. Wheezing rhinovirus illnesses in early life predict asthma development in high-risk children. Am J Respir Crit Care Med 2008; 178: 667-672.

14 Kusel MM, de Klerk NH, Kebadze T, et al. Early-life respiratory viral infections, atopic sensitization, and risk of subsequent development of persistent asthma. J Allergy Clin Immunol 2007; 119: 1105-1110.

15 Hyvarinen MK, Kotaniemi-Syrjanen A, Reijonen TM, et al. Teenage asthma after severe early childhood wheezing: an 11year prospective follow-up. Pediatr Pulmonol 2005; 40: 316-323.

16 Valkonen H, Waris M, Ruohola A, et al. Recurrent wheezing after respiratory syncytial virus or non-respiratory syncytial virus bronchiolitis in infancy: a 3-year follow-up. Allergy 2009; 64: 1359-1365.

17 Lehtinen P, Ruohola A, Vanto T, et al. Prednisolone reduces recurrent wheezing after a first wheezing episode associated with rhinovirus infection or eczema. J Allergy Clin Immunol 2007; 119: 570-575.

18 Midulla F, Pierangeli A, Cangiano G, et al. Rhinovirus bronchiolitis and recurrent wheezing: 1-year follow-up. Eur Respir J 2012; 39: 396-402.

19 Koponen P, Helminen M, Paassilta M, et al. Preschool asthma after bronchiolitis in infancy. Eur Respir J 2012; 39: 76-80.

20 Wainwright $C$. Acute viral bronchiolitis in children - a very common condition with few therapeutic options. Paediatr Respir Rev 2010; 11: 39-45.

21 Blom D, Ermers M, Bont L, et al. Inhaled corticosteroids during acute bronchiolitis in the prevention of post-bronchiolitic wheezing. Cochrane Database Syst Rev 2007; 1: CD004881.

22 Zhang L, Mendoza-Sassi RA, Wainwright C, et al. Nebulized hypertonic saline solution for acute bronchiolitis in infants. Cochrane Database Syst Rev 2008; 4: CD006458.

23 Cane RS, McKenzie SA. Parents' interpretations of children's respiratory symptoms on video. Arch Dis Child 2001; 84: 31-34.

24 Gern JE. The ABCs of rhinoviruses, wheezing, and asthma. J Virol 2010; 84: 7418-7426.

25 Simmonds P, McIntyre C, Savolainen-Kopra C, et al. Proposals for the classification of human rhinovirus species $C$ into genotypically assigned types. J Gen Virol 2010; 91: 2409-2419.

26 Singh AM, Moore PE, Gern JE, et al. Bronchiolitis to asthma: a review and call for studies of gene-virus interactions in asthma causation. Am J Respir Crit Care Med 2007; 175: 108-119.

27 van der Zalm MM, Uiterwaal CS, Wilbrink B, et al. The influence of neonatal lung function on rhinovirus-associated wheeze. Am J Respir Crit Care Med 2011; 183: 262-267.

28 Copenhaver CC, Gern JE, Li Z, et al. Cytokine response patterns, exposure to viruses, and respiratory infections in the first year of life. Am J Respir Crit Care Med 2004; 170: 175-180.

29 Contoli M, Message SD, Laza-Stanca V, et al. Role of deficient type III interferon-lambda production in asthma exacerbations. Nat Med 2006; 12: 1023-1026.

30 Gern JE, Rosenthal LA, Sorkness RL, et al. Effects of viral respiratory infections on lung development and childhood asthma. J Allergy Clin Immunol 2005; 115: 668-674. 